\title{
The Simulation Model of a Student Restaurant
}

\author{
Zvonimir Mihaljevic, Goran Dukic*
}

\begin{abstract}
This paper features a simulation model of the FSB canteen. The data required for the simulation was collected through direct observation of the canteen during the busiest time of the day, that is, lunchtime. The simulation model was verified and validated based on the collected data. The model and the results of the simulation were presented. In order to reduce the waiting time, two modifications of the existing model were proposed and simulation models for both cases were also developed. After simulating the modified models, in order to determine the effects of the proposed modifications, the obtained results were analyzed and compared with the results of the existing system.
\end{abstract}

Keywords: model; modification; simulation; validation; verification

\section{INTRODUCTION}

When a demand for a service exceeds the ability of a provider to respond to orders, a waiting line occurs. The set of statistical laws and theorems that deal with various problems related to the clients waiting for a service is called Queuing theory. Queuing theory is a mathematical study of the congestion and delays of waiting in line. "Queuing theory (or "queueing theory") examines every component of waiting in line to be served, including the arrival process, service process, number of servers, number of system places, and the number of customers which might be people, data packets, cars, etc." [1]. Waiting in line at the canteen is a part of every student's daily routine. Generally, students have limited periods of free time which they use to go to the canteen. The length of the queue often determines whether they decide to wait or decide it is not worth waiting for. Waiting in line is perceived negatively by students and is associated with poor service quality. Interestingly, the queues often occur when the system is not actually overloaded, that is when its capacity is not fully utilized. The main problem is in the patterns of arrivals and the process of serving; they are very variable and can cause a temporary overload on the system. The aim of this paper is to analyze the operation of the canteen as a multichannel system. The paper is derived from the master diploma work. Chapter 2 is followed by a brief theoretical background about simulations and simulation modelling. The third chapter presents the steps of a simulation project through the FSB canteen (FSB - Fakultet Strojarstva i Brodogradnje = FMENA - Faculty of Mechanical Engineering and Naval Architecture, University of Zagreb, Croatia). In order to get the most accurate simulation, before even creating a simulation, it is necessary to analyze the system in detail and measure all of its essential elements. Later on, with the help of all of those elements, we will improve the system and ultimately reduce the time spent waiting in line.

\section{SIMULATION AND SIMULATION MODELLING}

According to Meng [2], simulation is the imitation of the operation of a real-world process or system over time. The steps of a simulation process include:

- Generating an artificial history of a system.
- Observing the behavior of that artificial history.

- Drawing inferences concerning the operating characteristics of the real system.

Main advantages of simulation include:

- Studying the behavior of a system without building it.

- Results are accurate in general and compared to the analytical model.

- It helps to find an unexpected phenomenon in behavior of the system.

- It is easy to perform "What-If" analysis.

Main disadvantages of simulation include:

- It is expensive to build a simulation model.

- It is expensive to conduct a certain simulation.

- Sometimes it is difficult to interpret the simulation results.

\subsection{Systems and System Environment}

According to [2], a system is a group of objects which are joined together in some form of a regular interaction or interdependence towards the accomplishment of a certain purpose. A system is often affected by changes occurring outside of it. Such changes are said to occur in the system environment. In modelling a system, it is necessary to decide on the boundary between the system and its environment. System can be both discrete and continuous. A discrete system is one in which the state variable(s) change only at a discrete set of points in a certain time period. A continuous system is one in which the state variable(s) change continuously over time. The system consists of the following components:

- An entity is an object of interest in the system.

- An attribute is a property of an entity.

- An activity represents a time period of a specified length.

- The state of a system is defined as a collection of variables necessary to describe the system at any time, and relative to the objectives of the study.

- An event is defined as an instantaneous occurrence that may change the system's state. 
As it evolves over time, the behavior of a system is being studied through developing a simulation model.

\subsection{Modelling}

According to Basch and Žagar [3], modeling is the process of describing a model in a simulation language. In doing so, the actual system is displayed in a way enabled by the simulation language. There are different languages for different types of systems. A model is a means by which we represent a realistic system. The model only shows the most important aspects of the system, that is, the model describes the system only with a certain level of accuracy. In this paper, to create a simulation model of a student restaurant, Enterprise Dynamics 10.3. is used. Enterprise Dynamics represents a state of the art modular object oriented simulation platform created to help solve any complex people, processes, technology, and infrastructure related challenges. Moreover, Enterprise Dynamics offers datadriven answers for the majority of commercial, governmental, education and industrial applications [4]. The steps of making a simulation model of the FSB canteen are presented in next chapter.

\section{THE STEPS OF MAKING A SIMULATION MODEL OF THE FSB CANTEEN}

\subsection{Operation Flow}

In order to describe the real system and give us a rough picture of what the simulation model should look like, a conceptual model was created prior to the simulation model. The conceptual model of the canteen is shown in Fig. 1.

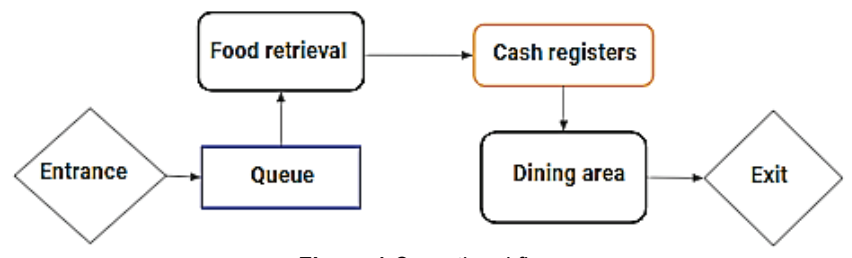

Figure 1 Operational flow

\subsection{Data Collection}

The input data for the canteen simulation was obtained on the basis of measurements performed during the canteen operation. Using a stopwatch to record the operation of the system, the data was collected through direct observation of the system. The description of the system required measurements of:

- arrival time of each student,

- time necessary to receive food,

- a possible number of students in a queue,

- a number of cash registers,

- invoicing time,

- a possible number of students in a queue for each cash register,

- dining time,

- canteen capacity,

- tray disposal time,
- a number of students entering the canteen at the opening.

Measurement of inter-arrival time consisted of noting the time whenever a new student joined the queue. This measurement also obtained the total number of students who came to the canteen during that particular day. A sample of 50 students was chosen to measure certain elements such as the amount of time for students to pick up food, for the cashier to issue an invoice, and the amount of time for student to pay for the meal. When measuring dining time, a sample of 100 students was taken. The amount of time students spend eating was measured. While measuring this amount of time, it was noticed that students who came to the canteen on their own, left it much faster, unlike the groups of students of three or more who tend to stay in the canteen longer despite finishing their lunch. In the end, for the measurement of tray disposal time, a sample of 50 students was taken. The amount of time for the canteen staff to pick up and clean the trays with plates and cutlery was measured. During this process of staff cleaning up the trays, especially during the busiest time in the canteen, a queue of students waiting tends to create. Furthermore, in theory, such a queue can cause a standstill, but it does not happen in practice. As a result, the type of measurement concerning the amount of time for the staff to clean the trays was chosen.

\subsection{Simulation Model of FSB Canteen}

Based on the conceptual model and a detailed observation of the canteen operation, a simulation model of the FSB canteen was developed in the Dynamics Enterprise simulation program. Fig. 2 shows the overall model used to analyze the FSB canteen operation.

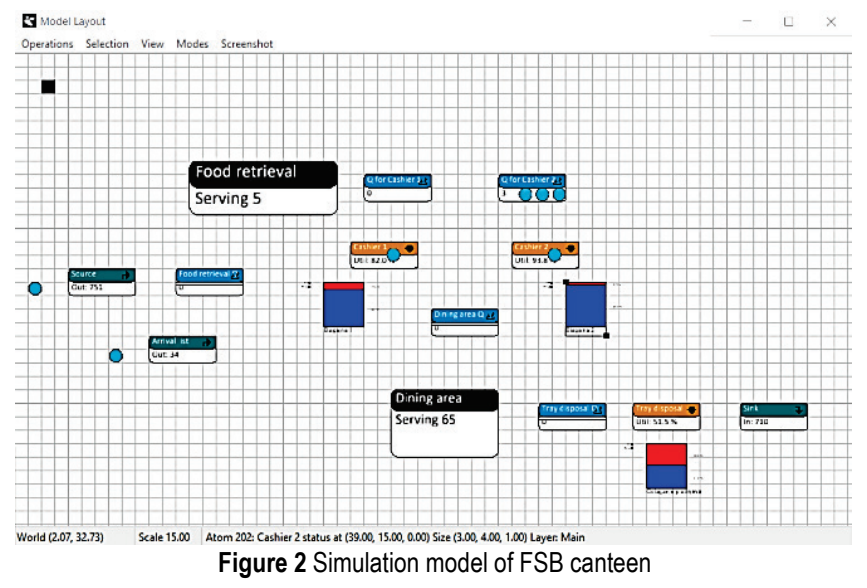

The basic atoms used in this model are Source, Queue, Server, Multiserver and Sink atoms. The simulation was conducted through a time period of three hours, that is, during lunchtime from 11:00 to 14:00, a time period when the system is at its busiest. The measurement results described in the previous chapter were used as input data to simulate the operation of the canteen. In order to capture the variability of the data, AutoFit option was used. The option was also used to find the probability distributions that best fit the observed data. The Source atom, which on average takes 14.25 seconds 
to perform its activity, generates students. Alongside the Source atom, Poisson distribution was assumed. Arrival list generated 34 students who initially loaded the system. When entering the canteen, students may have to wait in a queue before being served in Food retrieval atom. The average time required for students to pick up their food is 73.39 seconds for which the Logistic distribution fitted the data best. According to a random open channel rule, Multiservice Food retrieval chooses a random channel from all the open output channels and sends student to it. Then, students approach one of the cash register queues from which they go to the corresponding cash register. An average time for the first cashier to issue an invoice is 22.75 seconds for which the normal distribution fitted the data best. An average time for the second cashier is 26.25 seconds and pearsont5 distribution fitted the data best. After students pay for their food, they go to Dining area. An average dining time is 922.34 seconds for which an AutoFit suggests a Gamma distribution. When students finish their lunch, they are required to return the trays with plates and cutlery. An average time for an employee to clean a tray is 7.77 seconds for which the lognormal distribution fitted the data best.

\subsection{Verification and Validation of the Simulation Model}

According to [5], verification is a process which ensures that the model is constructed correctly, that is, it raises the question whether the mathematical model is correctly implemented in the simulation software or not. Verification also takes care of the correctness of the input parameters and logical structure of the model. Validation is a process of evaluating the accuracy of a model relative to the actual system it describes.

\subsubsection{Verification}

The simulation model is verified by investigating whether the model is built correctly. The model in Fig. 2 is built according to specifications by implementing the observed data. After running the model for three hours no errors occurred and all the students who had arrived at the canteen were served and eventually left the canteen. During this period of time, all atoms behaved as they were programmed.

\subsubsection{Validation}

This model was validated based on the data of the total number of students who arrived at the canteen the day the measurement was carried out. According to the canteen staff, 811 invoices were issued during lunchtime, while simulation generated 796 students during a three-hour period. The number of 796 students was obtained through the sum of students which was generated both by the atom Source (762 students) and students from the Arrival list (34 students). The comparison of the data obtained from the canteen staff ( 811 students in the canteen that day) and the simulation (796 students generated), validates the simulation model.

\subsection{Simulation Results of the Current System}

This section presents the results obtained by running the simulation model for a three-hour period for which certain measurements were performed. In the Run Control menu, the simulation is set to stop after three hours. Recording was turned on so simulation results could be read from the summary report. Fig. 3 shows the output of the summary report for the simulation model of the current system.

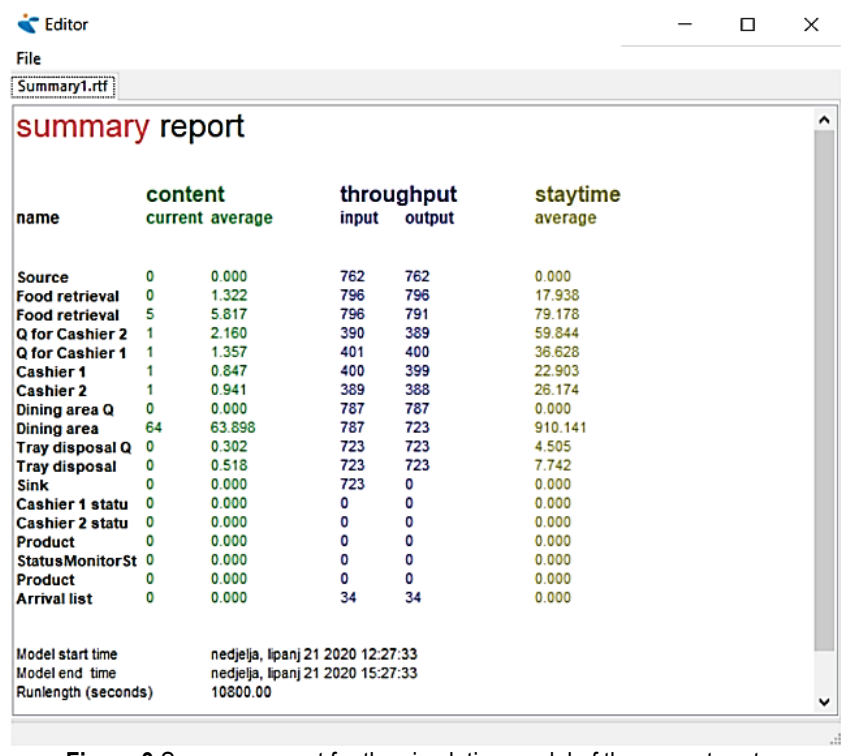

Figure 3 Summary report for the simulation model of the current system

The calculated utilization of the current system is 89.45 $\%$. This was calculated using the arithmetic mean of the utilization of Cashier $1(84.7 \%)$ and Cashier $2(94.2 \%)$. The average time spent in the system (W) obtained by the simulation is 169.89 seconds. The calculated time spent in the system (W) includes all elements of the system placed in front of the Dining atom in the model and as such is taken into account in further analysis. Although this particular system does not include all elements of a regular system, it is still called a system because it includes the elements such as the time spent in queues, the time spent in the multiserver Food retrieval, and the time spent on servers (cash registers). The period of time a student spends eating is not affected by any part of the system, but is defined by the students themselves. As a result, the element of dining time is not included in the analysis. The time spent in the tray disposal queue is negligible compared to the total amount of time spent in the system. Since that amount of time can be compensated for the dining time reduction, it is also not used in further analysis.

\section{SYSTEM MODIFICATIONS}

After the system's operational characteristics were obtained by simulating the canteen operation, certain modifications of the system were made in order to obtain more favorable values of such characteristics. Those modifications of the system include adding the third cash register and also adding another waiting line. Both system 
modifications are feasible in reality and they have both yielded some improvements in the system's operational characteristics. After that, a "What-if" analysis of the system was performed. In such an analysis the behavior of all three models in relation to the increased system load was analyzed.

\subsection{3-Cash Register System}

Fig. 4 shows a model of the canteen with three cash registers.
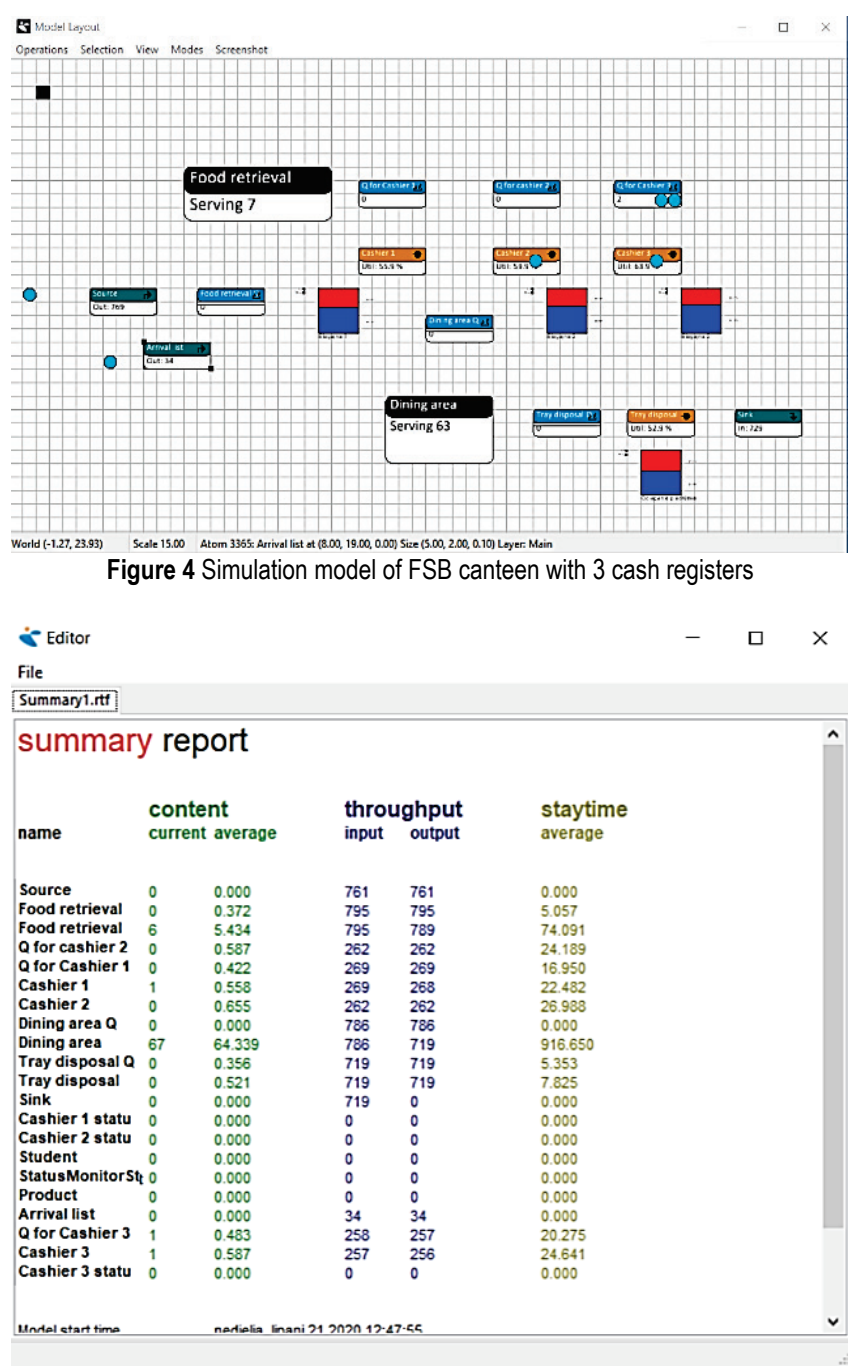

Figure 5 Summary report for the three-cash register canteen system

Unlike the initial model, this one has two additional atoms: Server (Cashier 3) and Queue (Queue for third cashier). Multiserver Food retrieval now has 3 output channels that are connected to the queues of each cash register. Random channel rule randomly selects the channel and outputs the Product (student) to it. After waiting in one of the queues, the student goes to the next cashier and further into the system that has remained the same. The simulation was also conducted throughout a period of three hours, for which the results are shown in Fig. 5.

By simulating a three-cash register canteen system, the system utilization of $60 \%$ was obtained. The total efficiency of $60 \%$ was obtained by the arithmetic mean of the usability of Cashier 1, Cashier 2, and Cashier 3. The average amount of time spent in the system (W) obtained by the simulation is 124.323 seconds.

\subsection{2-Row Waiting Line System}

Fig. 6 shows a two-row waiting line system.

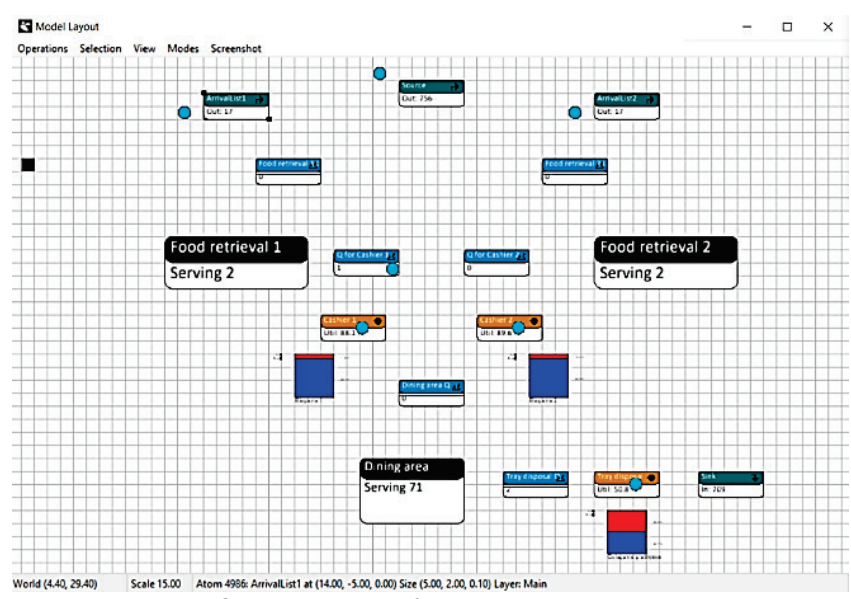

Figure 6 Simulation model of the two-row waiting line system

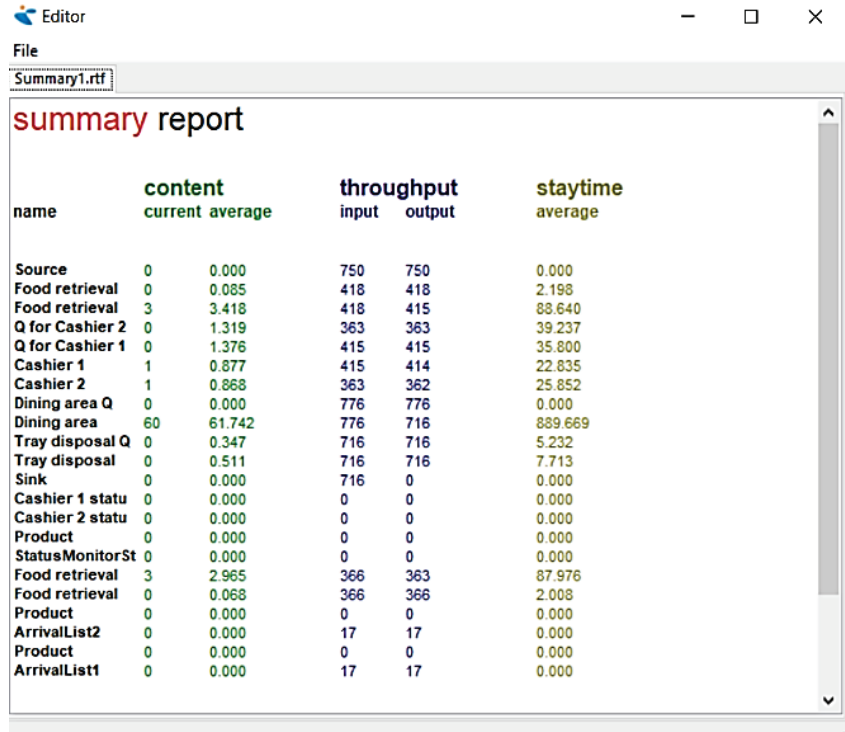

Figure 7 Summary report for the two-row waiting line system

Unlike the initial model, this model has two Food retrieval Multiservers, where each one has its own queue. Atom Source now has two output channels and randomly selects the queue to which the Product (student) is sent. It was assumed that out of 34 students who loaded the system at the beginning, half of them would fit in one row and the other half would fit in another one. This is done by adding another Arrival list to the system. The simulation was performed throughout the period of three hours for which the results are shown in Fig. 7.

System utilization of the current system was calculated at the percentage of 87.25. This was calculated using the arithmetic mean of the utilization of Cashier $1(87.7 \%)$ and 
Cashier 2 (86.8\%). The average amount of time spent in the system $(W)$ obtained by the simulation is 152.273 seconds.

\subsection{Model Comparison}

Tab. 1 shows a comparison of the system utilization and the average time spent in the system for all three developed models.

Table 1 Comparison of simulation model results

\begin{tabular}{|l|c|c|}
\hline & $\begin{array}{c}\text { System } \\
\text { utilization, \% }\end{array}$ & $\begin{array}{c}\text { Average time spent in } \\
\text { the system, } \mathrm{s}\end{array}$ \\
\hline Current system & 89.45 & 169.89 \\
\hline three-cash register system & 60 & 124.323 \\
\hline two-row waiting line system & 87.25 & 152.273 \\
\hline
\end{tabular}

The average waiting time in the three-cash register system is 124.323 seconds, which is almost $27 \%$ less than in the existing system. The system utilization was also reduced by 29 percentage points. The average waiting time in the tworow waiting line system is 152.273 seconds, which is $10 \%$ less than in the existing system. Compared to the current system, the system utilization reduced by two percentage points. Looking at the comparison, the high utilization of the system can be directly related to the higher average of waiting time. As a result, it can be inferred that the cash registers are the bottleneck of the system. Analyzing the simulation of the current system, a slight increase in the number of students coming into the system is visible. After the system suffers an initial shock where 34 students instantly load the system, Multiserver Food retrieval rarely reaches maximum capacity since queues are regularly filled before reaching the cash registers. As the number of students coming into the system increases, the usability of the cash registers reaches $99 \%$ very quickly with a result of endless queues starting to form. Based on this observation, it can be concluded that cash registers are the bottleneck of the system.

\section{4 "What-If" system analysis}

In order to reveal the bottleneck of the system mentioned in the previous subchapter, an analysis of the model which observes what happens if the number of students coming to the canteen increases was made. "What-if" analysis is an analysis that looks at what would happen to the system if any of its parameters changed. The following table shows what happens to all the three systems if the number of students coming to the canteen increases by $10 \%$, that is, how this increase affects the usability of the system and the average time students spend waiting in the system.

Increase in the number of students coming to the canteen by $10 \%$ results in significant changes of characteristics inside the two systems. The current system and the two-row waiting line system, which have been heavily burdened so far, show that they are able to withstand such an increase in the number of students. As a result, this kind of increase also causes a sharp increase in the average waiting time in the system. In the current system, there was an increase of $41 \%$ in the waiting time, while in the system with two rows, the increase was $33 \%$. The three-cash register system withstood this increase very well. Its utilization increased only by 4.23 percentage points and the average time spent in the system increased only by $2.35 \%$. The results show that adding another cash register to the system has a greater potential than adding another waiting line. The three-cash register system withstood an increase without any problems and with a minimal increase in the average time spent in the system. Further testing showed that this system is able to handle up to $40 \%$ more students than the current system, without compromising its operational characteristics.

Table 2 Results comparison of the "What-lf" analysis

\begin{tabular}{|l|c|c|}
\hline & $\begin{array}{c}\text { System } \\
\text { utilization, } \%\end{array}$ & $\begin{array}{c}\text { Average time spent in } \\
\text { the system, } \mathrm{s}\end{array}$ \\
\hline Current system & 98.50 & 287.18 \\
\hline three-cash register system & 64.23 & 127.32 \\
\hline two-row waiting line system & 95.45 & 227.69 \\
\hline
\end{tabular}

\section{CONCLUSIONS}

In order to analyze the work of the FSB student canteen and to find out how much time students spend waiting in line at the canteen, canteen simulation model was created with the help of the Enterprise Dynamics simulation platform. The data used in the simulation was obtained through direct observation of the system during lunchtime when the number of students visiting the canteen is the greatest. The high accuracy of the simulation results enabled further modifications of the model with the aim of optimizing the whole process, and ultimately reducing the average time spent in the system. It can be concluded from the obtained results that both modifications offer an improvement in terms of the average waiting time in the system. However, the addition of a third cash register in the system has a greater potential. Carrying out a "What-if" analysis leads to the conclusion that the three-cash register system is able to handle up to $40 \%$ more students than the existing one, without compromising operational characteristics. As a result, serious consideration should be given to reconstructing the existing system.

\section{Notice}

The paper was presented at MOTSP 2020 - International Conference Management of Technology - Step to Sustainable Production, which took place from $30^{\text {th }}$ September $-2^{\text {nd }}$ October 2020 in Bol, island Brač (Croatia). The paper is not and will not be published anywhere else.

\section{REFERENCES}

[1] https://www.investopedia.com/terms/q/queuing-theory.asp

[2] Meng, X. (1998). Simulation CSCI 6337. University of Texas - Pan American, https://www.eg.bucknell.edu/ xmeng/ Course/CS6337/Note/master/master.html

[3] Basch, D. \& Žagar, M. (2011). ATLAS - simulacije arhitekture mikroračunala, 1. internetsko izdanje. Sveučilište u Zagrebu, Fakultet elektrotehnike i računarstva, Zagreb. http://docbook.rasip.fer.hr/ddb/public/index.php/publication/h $\mathrm{tml} / \mathrm{rasipbook} / \mathrm{id} / 3$ ? chapter $=$ TOC $\&$ rce $=0 \& \mathrm{tts}=0 \& \mathrm{css}=$ original \&edit $=0$ (in Croatian) 
[4] https://www.incontrolsim.com/software/enterprise-dynamics/

[5] Banks, J., Carson II, J. S., Nelson, B. L., \& Nicol, D. M. (2005).

Discrete-Event System Simulation, $4^{\text {th }}$ edition. Prentice Hall.

\section{Authors' contacts:}

Zvonimir Mihaljevic, mag. ing. mech.

University of Zagreb,

Faculty of Mechanical Engineering and Naval Architecture,

Ivana Lučića 1, 10000 Zagreb, Croatia

E-mail: zvone.miha@gmail.com

Goran Dukic, PhD, Full Professor

University of Zagreb,

Faculty of Mechanical Engineering and Naval Architecture,

Department of Industrial Engineering, Chair of Production Design,

Ivana Lučića 1, 10000 Zagreb, Croatia

Tel. +385 1/6168381

E-mail: goran.dukic@fsb.hr 